\title{
Zetri - the cereal of future?
}

Sokolov V.A.

Institute of Molecular and Cellular Biology, SB RAS, Russia

e-mail: sokolov@mcb.nsc.ru

The most common cereal in the 20th century was maize (Zea mays ssp. mays, $2 n=$ $=2 x=20$ ). In the 21 st century, its production in the world reached 1 billion tons and continues to increase annually. First of all, this is due to the high adaptability of its cultivation and the fact that being a $\mathrm{C}_{4}$ plant of the photosynthesis pathway, it most effectively transforms solar energy into plastic substances. One of the problems associated with its cultivation, is the need for annual production of F1 hybrids, because they are more productive. For a long time, researchers tried to consolidate heterosis by transferring the corn to a cornless way of reproduction from a wild relative, the gamagrass (Tripsacum dactyloides, $2 n=4 x=72$ ). However, contrary to expectations, this trait was under complex genetic control and its expression requires the presence of 9 chromosomes of the wild relative, therefore, the resulting plants are far from maize.

In this regard, we propose to create apomictic 56-chromosomal maize hybrids with gammagrass $(2 n=4 x=56=20 \mathrm{Zm}+36 \mathrm{Td})$. As the source of the genomes from maize, the maize lines used in hybrid selection for heterosis in F1 are taken. At present, such hybrids have been obtained, but they are male-sterile, and forced pollination of corn is required to obtain seeds. Nevertheless, we believe that these hybrids have so many beneficial economic traits that the work of identifying pollen-producing plants among them is justified and should be expanded and continued. First of all, these hybrids are salt tolerant and do not suffer from short-term waterlogging, and are also resistant to many infectious diseases and other biotic pests. Since the maize genome is unusually dynamic, the successful breeding improvement of such hybrids is fully justified. 